\title{
BIM Management Guidelines of the Construction Process for General Contractors
}

\author{
Salvatore Viscuso, Cinzia Talamo, Alessandra Zanelli and Ezio Arlati
}

\begin{abstract}
Capability of construction companies to manage the digital interoperability between stakeholders and technicians is fundamental for the success of a work, both for new constructions and renovations. The research outlines a methodological and operational protocol for general contractors and the related supply chains and subcontractors. The Programme contains indications for the development of BIM-based workflows that implement the main activities in building construction, operations and maintenance: off-site and on-site time schedules, site planning and building ergotechnics, accounting, project variances, non-compliance issues, test and commissioning, maintenance planning.
\end{abstract}

Keywords Building information modeling $\cdot$ BIM protocol $\cdot$ Implementation plan • Pilot project $\cdot$ Building construction $\cdot$ Facility management

\section{Introduction}

Building Information Modeling (BIM) is a process focused on the development, use and transfer of a digital information model of a building project to improve the design, construction and operations of a project or portfolio of facilities. When properly implemented, BIM can provide many benefits to a project. Well-planned projects illustrate the value of BIM that yield: increased design quality through effective analysis cycles; increased innovation using digital design applications; greater prefabrication due to predictable field conditions; improved field efficiency by visualising the planned construction schedule. To improve the overall performance of the facility or a portfolio of facilities, operators in asset management, space planning and maintenance scheduling can use valuable information obtained at the end of the construction phase. Yet, there are also examples of projects where the company's technical operators - hereinafter referred to as the "team"- - did not effectively plan

S. Viscuso $(\bowtie) \cdot C$. Talamo $\cdot$ A. Zanelli $\cdot$ E. Arlati

Architecture, Built Environment and Construction Engineering-ABC Department, Politecnico di Milano, Milan, Italy

e-mail: salvatore.viscuso@polimi.it

B. Daniotti et al. (eds.), Digital Transformation of the Design, Construction and Management Processes of the Built Environment, Research for Development, https://doi.org/10.1007/978-3-030-33570-0_17 
the implementation of BIM and incurred increased costs for the modeling services, schedule delays due to missing information, and little to no benefit. Implementing BIM requires detailed planning and fundamental process modifications for the project team members to achieve the value from the available model information (Eastman et al. 2011).

Currently the difficulties of a successful integration between BIM processes and production are directly related to the number of participants in the project. If, in architecture, the contemporary trend is to design building with technological complexity that distinguishes it as an identifying icon or landmark, the difficult task of managing a huge amount of information data and requirements in building construction and operations, often impossible to systematise if not through a predefined organizational approach, represents a challenge (Ciribini 2016).

Furthermore, in order to achieve a real synergy between the interoperable BIM design and the production fields, it is necessary to organise the work-sharing using cloud technologies; this necessary produces the application of standardised, secured rules to protect shared project milestones and work progresses.

To integrate BIM into the project delivery process, it is important to develop a detailed execution plan for companies involved in construction and asset management. Within the activities of a research contract with Pessina Costruzioni SpA, an Italian General Contractor specialised in construction and operations of healthcare facilities, the Department of Architecture, Built Environment and Construction Engineering (DABC) of Politecnico di Milano has developed a BIM Protocol that outlines the overall vision along with implementation details for construction and operations.

This Protocol provides a structured procedure for creating and implementing a corporate BIM Project Execution Plan that cover the main business activities of the company. The four steps within the procedure include:

1. the identification of high-value BIM uses during project planning, design, construction and operational phases;

2. the design of tailored BIM execution process by creating process maps;

3. the definition of BIM deliverables in the form of information exchanges;

4. the development of the infrastructure in the form of contracts, communication procedures, technology and quality control to support the implementation.

Specified sections of the BIM Protocol explain the details related to each step. Detailed templates have also been created to support each of these steps. These templates are included in the Appendices of the document.

The Protocol is compliant with the following standard: Industry Foundation Classes (IFC), defined by UNI EN ISO 16739:2016; UNI 11337:2017 (Building and civil engineering works-Digital management of the informative processes) that regulates interoperability between project objectives and digital modeling; BS EN ISO 19650:2018 (Organization and digitization of information about buildings and civil engineering works, including building information modelling-Information management using building information modelling). 


\section{Identify BIM Goals and Uses}

The preparation of the process has as its first major step the definition of the project uses in relation to main Contractor's works. Their identification is fundamental, as it made possible defining the final objectives, correctly setting the models and identifying the parameters that allow the optimization of the scenario that best meets the purposes. An incorrect setting of the requirements can nullify all the modeling work done later (Deutsh 2011).

In accordance with a typically top-down decision-making process, a series of macro-categories define the criteria to evaluate projects, including the budget, the technical-construction feasibility, the internal distribution and the energy performances. The definition of these is carried out in close collaboration with the team and through some briefings involving suppliers and sub-contractors.

Once the team has defined measurable goals, both from a project perspective and company perspective, then the specific BIM uses on the project can be identified. The Protocol includes a list of common uses for BIM, which have been identified through analysis of project case studies, interviews with industry experts, and review of the literature. Each BIM use is a unique task or procedure on a project that can benefit from the integration of BIM into that process. The list is not comprehensive but provides a good representation of the potential uses of BIM within a construction company. It includes design authoring, 4D modeling, cost estimating, space management and record modeling. The Contractor's team should identify and prioritise the appropriate BIM uses that they have identified as beneficial to the project goal achievement (Holzer and Downing 2010).

To facilitate the BIM use review process, a BIM Worksheet template includes a list of the potential BIM uses, along with fields to review the value, responsible party, capabilities, additional notes, and the decision from the team on whether to implement the BIM use.

\section{Design the BIM Execution Process}

Once the team has identified the BIM uses, a process mapping procedure can clarify all the progressive steps to implement traditional workflows. Initially, a high-level map showing the sequencing and interaction between the primary BIM uses on the project allows all team members to understand clearly how their work processes interact with the processes performed by other team members.

After the high-level map is developed, then team members responsible for each detailed BIM use should select or customise more detailed process maps. For example, the high-level map will show how energy evaluation, cost estimating, 4D modeling and recording are sequenced and interrelated. A detailed map will show the detailed processes that the Contractor can perform by itself or, in some cases, with 
the consultancy of external organizations such as designers, sub-contractors or, as may be the case for energy task, LEED certifiers.

Maps representing for each process should clearly identify responsible parties involved in the workflow. For some processes, this may be an easy task, but for others it may not. It is important in all cases to consider which team member is best suited to complete the task successfully. Additionally some processes may have multiple responsible parties. The identified party will be responsible for clearly defining the information required to implement the process as well as the information produced by the process (Fig. 1).

Referring to graphical notation and information format for the processes within the BIM overview maps, each process should include a process name, project phase, and the responsible party. Each process should also include the detailed map title for the process. This detailed map notation is necessary since several processes may

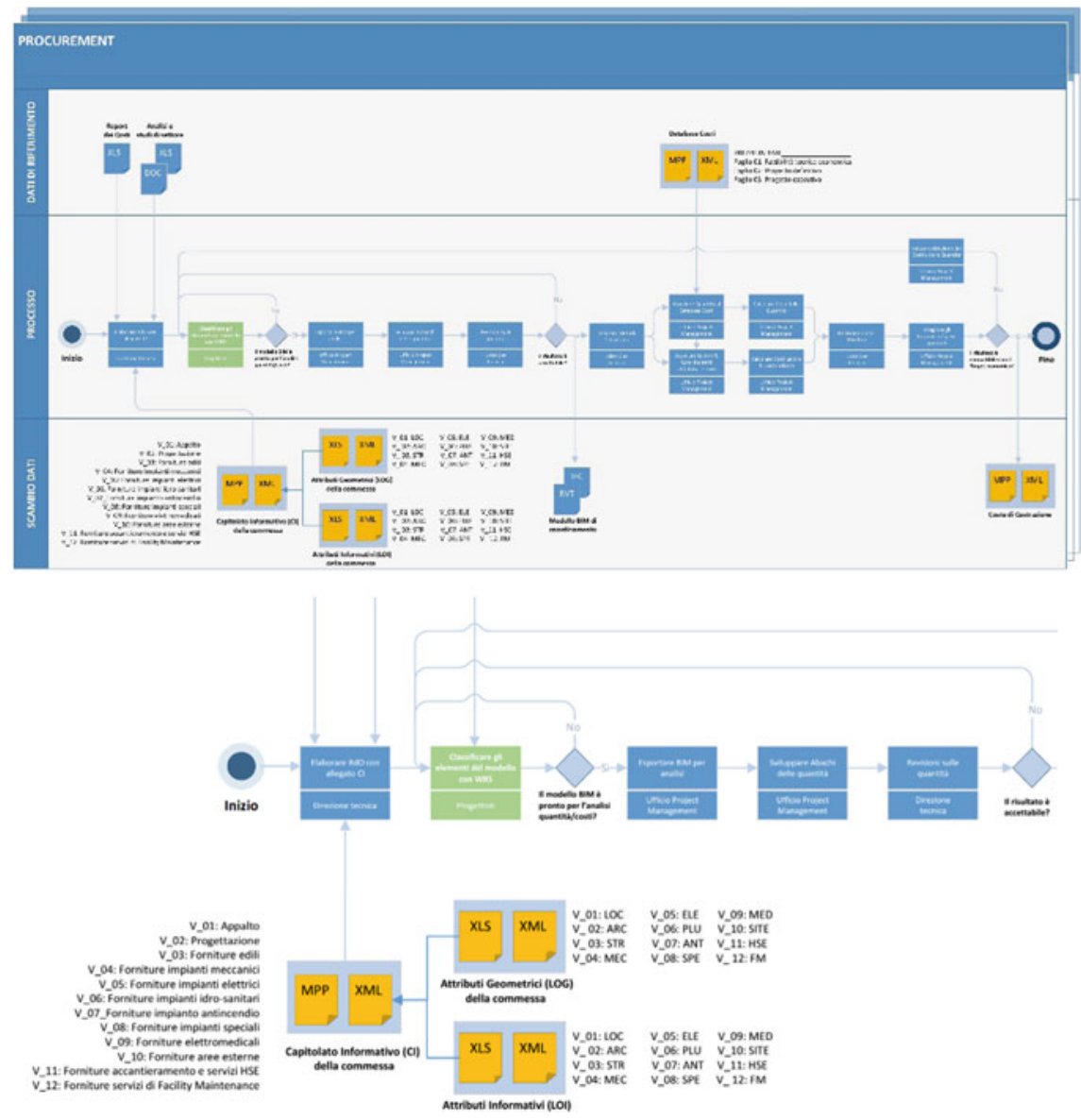

Fig. 1 Detailed process maps, organised in process, data exchange and reference data 
share the same detailed map. For example, a construction management company may perform cost estimating from the building information provided from the designer. The Construction manager may perform this estimate during the schematic design, design development and construction document phase, but it may utilise the same detailed workflow-obviously with diverse detail levels of BIM models, reference documents and templates- to accomplish this task, which can be represented in a single detailed map.

\section{Develop Information Exchanges}

The goal of this task is to present a method for defining information exchanges between project processes that are crucial to successful BIM implementation. To define these exchanges, the team needs to understand what information is necessary to deliver each BIM use. To assist in this task, an Information Exchange Worksheet template has been designed. The Contractors should complete the Information Exchange Worksheets in the early stages of a project, after designing and mapping the BIM process.

In compliance with Standard UNI 11337:2017 (Building and civil engineering works-Digital management of the informative processes), the definition of the information level through BIM parameters transfers project objectives to the digital models. For each information exchange transaction, it is important for the team members and, in particular, the author-designers, sub-contractors etc.-and receiver-the team-to understand clearly the information content. To define each information exchange, the following information should be documented:

1 Model Receiver: Identify all project team members that will be receiving the information to perform a future BIM use. These parties are responsible for filling out the Input Exchanges.

2 Model File Type: define the Level of Geometry (LOG) for each BIM use that can be associated to the project. The task also lists the specific software applications, as well as the version and the file format used to manipulate the model during each BIM use by the receiver. This is pertinent in order to identify any interoperability that may exist between exchanges.

3 Information: identify only the information necessary for the BIM use implementation (Fig. 2). Predefined property sets (P-Sets) organise the information parameters representing building elements and components. Parameter list changes depending on the Level of Information (LOI) needed for each BIM use. A proper BIM maturity level can interpolate the multidisciplinary skills of designers and technicians involved in the construction process. By sharing the P-sets in a common data environment (CDE), it will be possible to identify and solve overlaps and process interferences between different operators and designers. 


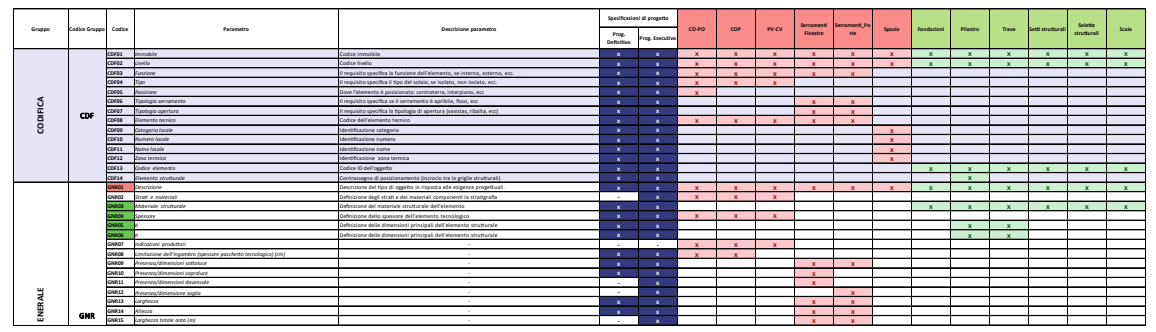

Fig. 2 Matrix parameters - building components, developed for schematic design, detailed design, construction design and as-built BIM models

\section{Define Supporting Infrastructure}

The final step of the BIM Protocol is to identify and define the project infrastructure required to implement effectively BIM as planned. Six specific categories support the BIM project execution process. After a deep evaluation of the literature review with the industry stakeholders and operators, the selected categories are Tender, Procurement, Construction, Recording, Asset Management and Quality Control.

Information for each category can vary significantly by project, therefore the goal of the description is to initiate discussion and address content areas and decisions that need to be made by the project team. Templates of Employer's Information Requirements (EIR) and BIM Execution Plan (Pre-contract and Post-contract) have been developed and shared with the team.

Quality control category is transversal to the different stages of the project. To ensure model quality in every phase and before information exchanges, procedures must be defined and implemented. Each BIM process activated during the lifecycle of the project must be pre-planned considering model content, level of detail, format and party responsible for updates; and distribution of the model and data to various parties. Each party contributing to the BIM model has a responsible person to coordinate the model that participates in all major BIM activities. He is responsible for addressing issues that might arise with keeping the model and data updated, accurate, and comprehensive.

\section{Pilot-Projects}

From January to December 2018 diverse pilot-projects involved the Contractor's team, in order to train and qualify the technicians that normally coordinate the company activities. The first project relates to the Public-Private Partnership proposal (PPP) for the construction of a novel Hospital Center in Lanciano (1st DEA level with 218 beds and net area of about 50,000 sm) in substitution of the obsoleted building. The proposal also included the Schematic design of the new hospital. The 
workflow started from the BIM uses definition, that involved in internal architects, structural and mechanical engineers and cost estimators. Modeling in separated files the diverse disciplines made it possible to adopt, in the definition phase of the sanitary space planning, a multicriteria strategy, that considered more options in the search for optimal solutions in function of predetermined weighted criteria (Viscuso et al. 2019).

Each health function was studied, in first analysis, evaluating all the interactions necessary within each department. This study therefore represented the basis for the development of a subsequent level of the individual departments' distribution. For defining different spatial and distribution scenarios and performing multicriteria analysis on them, indications provided by the facility managers guaranteed the compliance with the requirements needed for a possible Joint Commission Health Accreditation (Capolongo 2012). In order to evaluate the surfaces and volumes of this schematic design, the team interpolated the main data (surfaces, distances, airlight relationships) related to diverse design options and configurations: all medical and surgical specialties, diagnostic equipment and robotic equipment, laundry, sterilization, and catering. For a balanced pre-dimensioning, all human activities related to reception and waiting were also considered, such as commercial activities, bank, as well as all the babysitting services, gyms, etc. (Carpman and Grant 2016).

Discipline models were modeled separately at different Levels of Development. Initially three LOD 100 volumetric models of conceptual masses are created, useful for distributive analysis of three different design options, and for the verification of internal distribution specification (e.g. the separation between clean and dirty zones). Discipline models with LOD 200 were then realised, relating to the design option that was more in keeping with the health requirements (Fig. 3).

The second project consists in the development of the technical and economical documentation to be submitted for a construction bidding. The construction planning of the detail project of this tender-an 11-storey office building with net area of $27,000 \mathrm{sm}$ - has needed the implementation of 4D modeling, in order to simulate and verify the following construction strategies (Fig. 4).

The company's team composed the Day-work Programme through a logical succession of construction stages (Shell \& Core, Category A, Category B, Test \& Commissioning), minimising their temporal overlap and carefully assessing the impact on the site in terms of duration, management and safety. The logical-temporal connection between the different activities allows a "continuum" of the work and a high level of prefabrication off-site (Das and Kanchanapiboon 2011).

The above-explained organization was modelled by the company's team for 4D BIM use, starting from IFC models included in the bidding documentation. The tri-dimensional visualization of Work Schedule also performed a better construction management per each stage: in fact, a correct distribution of workers in specified sectors reduces and minimises interferences between different activities, e.g., avoiding contemporary overlaps into the same sector or floor (Zhang et al. 2016).

The third implementation has involved the restoration and the complete renovation of a Historic building located within the "Pontificio Istituto Missioni Estere" (PIME) Complex, which is in Milan. The building was built at the beginning of the last 

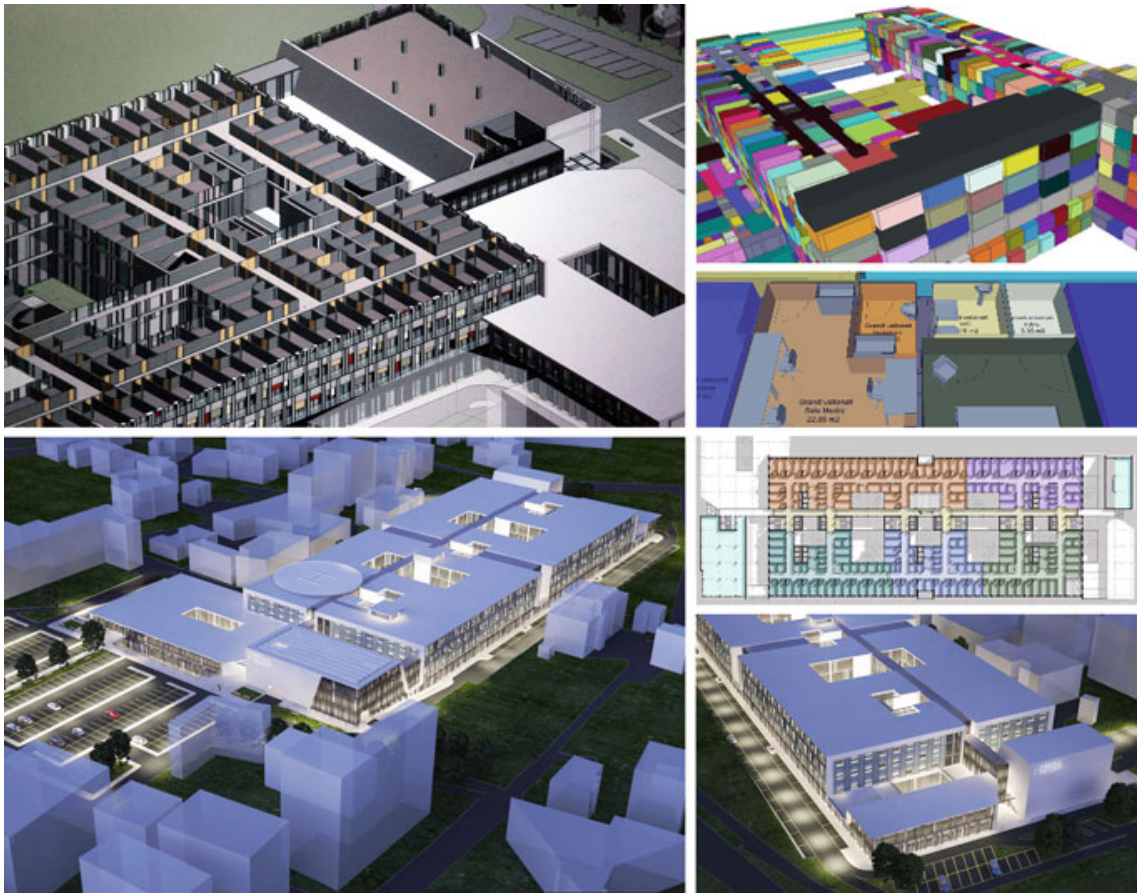

Fig. 3 ARC model of the new hospital center of Lanciano (LOD 200) and space planning optioneering (LOD 100)

century and is currently subjected to preservation by the Italian Superintendent of Archaeology, Fine Arts and Landscape. It consists of five floors for an overall net area of 5,857 sm. The detailed project-developed by General Planning Srl—designs commercial and exhibition functions at underground and ground levels, as well as offices and collective spaces and residences on the upper floors.

Within the pilot activities included in this research, the team have redacted a detailed Employer's Information Requirements (EIR) containing data, information and models to deliver at each construction progress report, together with the required Level of Definition. The purpose was to extract Shop drawings and Bills of quantity directly from BIM models per each Milestone, in order to provide to Procurement Office the exact information before authorising the purchasing of materials and services. Finally, the EIR contains the Level of Information to reach in the As-Built Model (LOD F) that will record the work as constructed and collect all the Building Log Book Documents (Fig. 5).

The last pilot project refers to the model check and the data population of BIM Models that are recently synchronised with the Facility Management software ARCHIBUS, in order to digitally drive operations and maintenance activities of the Ambulatory Care Centre "Navile" in Bologna. The set-up of web-based platform and the As-built modeling (Fig. 6) has been done by e-FM, company leader in the 

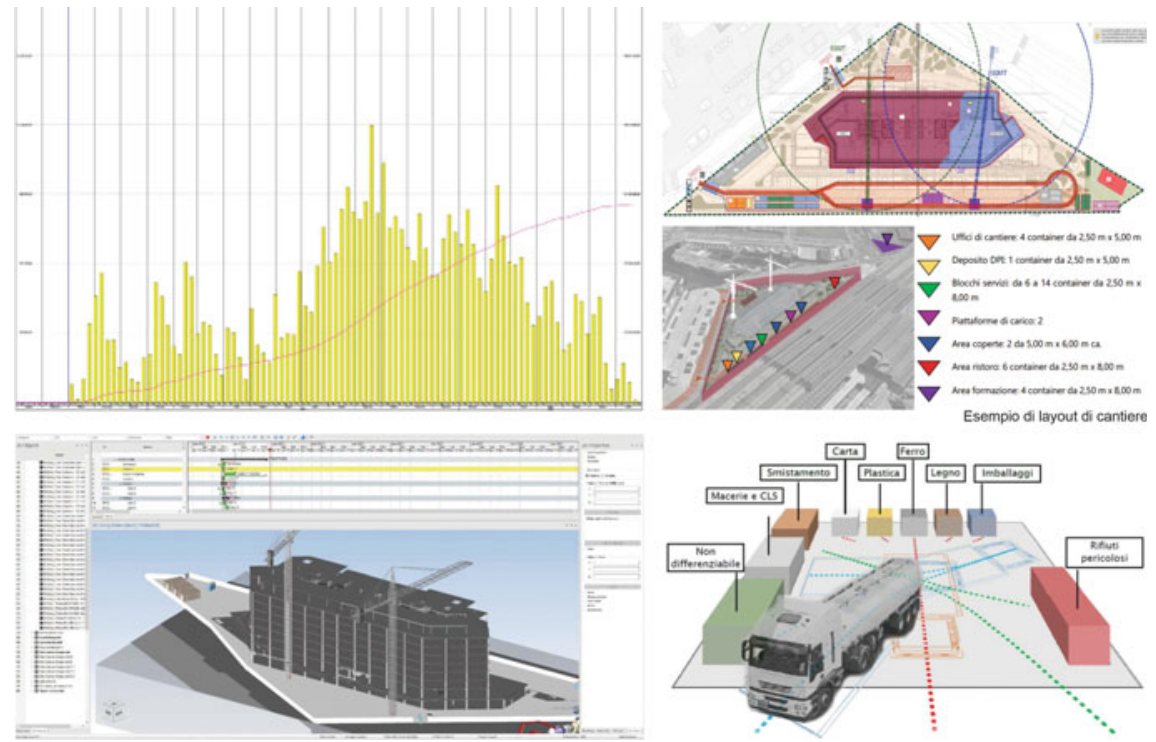

Fig. 4 Earned value analysis, 4D modeling and site layout are performed through the use of IFC models (LOD 350) included in the bidding documentation
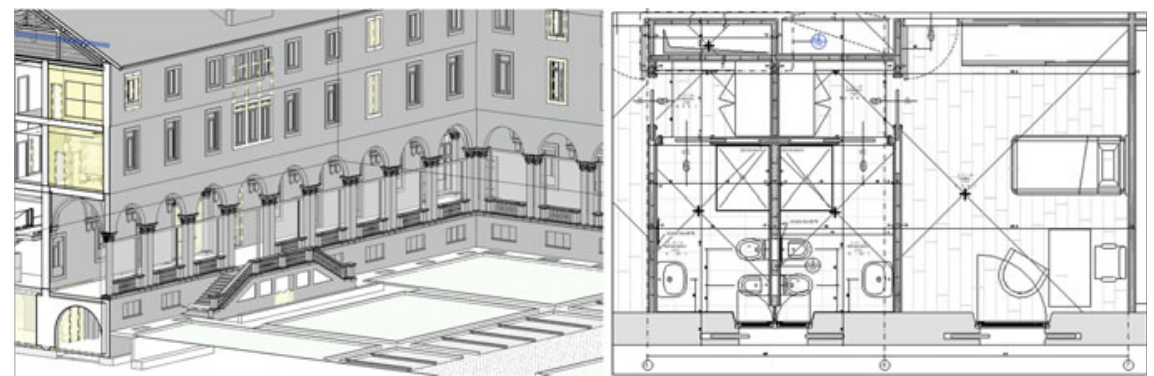

Fig. 5 Shop drawings — created with model authoring software — of PIME renovation
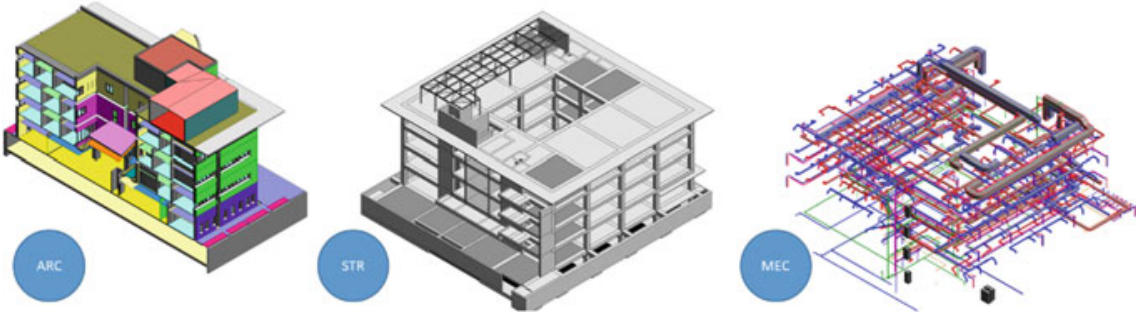

Fig. 6 As-built models of ambulatory care centre "Navile", organised per disciplines 
Asset digital management. In the short-term, the aim is that empower departments seamlessly coordinate how they share, use and maintain assets. In the long-term, it will be possible to create a plan that aligns capitals and maintenance budget with company's business goals, thus optimising operations for the whole asset lifecycle (Tronchin and Manfren 2015).

\section{Conclusion}

The experience under examination shows how the development of the BIM Plan into a complex organisation, such as a construction company and relative sub-contractors, is necessarily a collaborative process. The planning of a shared work methodology is an essential condition for obtaining a synergic collaboration between the team components. An underestimation in the planning of the process, or in the minimum requirements needed for the advancement to a subsequent state of work, can nullify the effort that involves the construction of the BIM models for separate disciplines, which would not have a return of investment in geometric modeling alone (Ciribini 2016). BIM can be implemented at specified phases throughout a project, but the current technology, training, and costs of implementation relative to real benefits must always be considered when determining the appropriate areas and levels of detail needed in the information modeling processes. Teams should not focus on whether or not to use BIM in general, but instead they need to define the specific implementation areas and uses. A team should aim to implement BIM at the level needed to maximise value while minimising the cost and impact of the modeling implementation (Arlati and Viscuso 2018). This requires the team to selectively identify appropriate areas for BIM implementation and plan these implementation areas in detail.

\section{References}

Arlati E, Viscuso S (2018) La modellazione digitale nel processo di intervento edilizio. In: Appalti Pubblici Riserve, Varianti, e Strumenti di Precontenzioso - Tipologie e Contenuti, Collana Norme \& Tributi, Il Sole 24 Ore, Milan, vol 9, pp 185-206

Capolongo S (2012) Architecture for flexibility in healthcare. Franco Angeli, Milano

Carpman JR, Grant MA (2016) Design that cares: planning health facilities for patients and visitors, 2nd edn. Jossey-Bass, San Francisco

Ciribini AL (2016) BIM e digitalizzazione dell'ambiente costruito. Grafill, Palermo

Das S, Kanchanapiboon A (2011) A multi-criteria model for evaluation design for manufacturability. Int J Prod Res 4(49):1197-1217

Deutsh R (2011) BIM and integrated design: strategies for architectural practice, 1st edi-tion. Wiley, New Jersey

Eastman C, Teicholz P, Sacks R, Liston K (2011) BIM handbook: a guide to building information modelling for owners, managers, designers, engineers and contractors, 2nd edn. Wiley, New Jersey 
Holzer D, Downing S (2010) Optioneering: a new basis for engagement between architects and their collaborators. Archit Des 80(4):60-63

Tronchin L, Manfren M (2015) Multi-scale analysis and optimization of building energy performance-Lessons learned from case studies. Procedia Eng 118:563-572

Viscuso S, Dragoljevic M, Zanelli A (2019) Analisi multicriterio per la progettazione preliminare di una struttura ospedaliera. In: Proceedings of SITdA 2018 international conference "La Produzione del progetto", Reggio Calabria (ongoing publication)

Zhang C, Zayed T, Hijazi W, Alkass S (2016) Quantitative assessment of building constructability using BIM and 4D simulation. Open J Civ Eng (6), 442-461

Open Access This chapter is licensed under the terms of the Creative Commons Attribution 4.0 International License (http://creativecommons.org/licenses/by/4.0/), which permits use, sharing, adaptation, distribution and reproduction in any medium or format, as long as you give appropriate credit to the original author(s) and the source, provide a link to the Creative Commons license and indicate if changes were made.

The images or other third party material in this chapter are included in the chapter's Creative Commons license, unless indicated otherwise in a credit line to the material. If material is not included in the chapter's Creative Commons license and your intended use is not permitted by statutory regulation or exceeds the permitted use, you will need to obtain permission directly from the copyright holder. 